\title{
MINIMALLY CLASSIFYING RELATIVE EQUILIBRIA
}

\author{
JULIAN I. PALMORE* \\ Dept. of Mathematics, The University of Michigan, Ann Arbor, Mich. 48109, U.S.A.
}

\begin{abstract}
Estimates on a minimal classification of relative equilibria in the planar $n$-body problem of celestial mechanics have been announced in [1], [2] . Our main theorem asserts that these estimates are actually met for any $n \geqq 3$ on an open set in $\mathbb{R}_{+}^{n}$. For any $n \geqq 4$, this open set is proper.
\end{abstract}

\section{INTRODUCTION}

In a preceding paper [2] we gave the setting of relative equilibria in the planar $n$-body problem. We study the set of critical points of a real analytic function $\widetilde{V}_{m}<0$ which is defined on a real analytic manifold $X_{m}$ where $n \geqq 3$ and $m=\left(m_{1} \ldots m_{n}\right) \in \mathbb{R}_{+}^{n}$ are fixed. The critical points of $\tilde{V}_{m}$ correspond in a 1-1 fashion with classes of relative equilibria.

For each $\left(m_{l}\right) \in \mathbb{R}_{+}^{n}$ we have that $X_{m}$ is homeomorphic to a Stein manifold $\mathbb{P}_{n-2}(\mathbb{C})-\widetilde{\Delta}_{n-2}$ where $\mathbb{P}_{n-2}(\mathbb{C})$ is complex projective space of (complex) dimension equal to $n-2$ and $\widetilde{\triangle}_{n-2}$ represents the configurations where two different masses coincide. $\widetilde{\triangle}_{n-2}$ is the union of $n(n-1) / 2$ codimension 1 complex projective subspaces. There is an involution on $X_{m}$ which corresponds to the action induced on $\mathbb{P}_{n-2}(\mathbb{C})$ by complex conjugation. This places a further restriction on the set of critical points of $\widetilde{V}_{m}$. It is well known that as a Stein manifold $\mathbb{P}_{n-2}(\mathbb{C})-\widetilde{\Delta}_{n-2}$ (hence $X_{m}$ ) has the homotopy type of a CW complex of (real) dimension $n-2$. The involution places on this $\mathrm{CW}$ complex the restriction that the cellular decomposition must be invariant under complex conjugation. Now $\widetilde{V}_{m}$ is a real analytic function with a compact critical set; therefore, by the main theorem of nondegenerate Morse theory (and a little work) we may always write

$$
\mathbb{P}_{n-2}(\mathbb{C})=\widetilde{\Delta}_{n-2} \cup e_{1} \cup \ldots \cup e_{r} \text { where } \operatorname{dim} e_{k} \geqq n-2
$$

and the standard notation for attaching a cell to a space is used.

It is an easy observation that this relative cellular decomposition of $\mathbb{P}_{n-2}(\mathbb{C})$ gives a partition of $\mathbb{P}_{n-2}(\mathbb{C})-\widetilde{\triangle}_{n-2}$ by open cells which are the interios of the cells $e_{1}, \ldots, e_{r}$. Furthermore, this relative decomposition of $\mathbb{P}_{n-2}(\mathbb{C})$ (or, the associated open partition of $\mathbb{P}_{n-2}(\mathbb{C})-\widetilde{\Delta}_{n-2}$ ) generates by duality the $\mathrm{CW}$ complex of (real) dimension $n-2$ with the same homotype type.

\footnotetext{
* Research supported in part by NSF Grant MCS 13-08818 A03.
} 
We conclude that by studying the $\mathrm{CW}$ complexes with the same homotype as $X_{m}$ (or, equivalently, the partitions of $X_{m}$ by open cells of dimension which equals or exceeds $n-2$ ) which are invariant under complex conjugation, we obtain the decompositions possibly given by $\widetilde{V}_{m}$ in the manner indicated above.

\section{MAIN THEOREMS}

In this section for any $n \geqq 3$, for any $k \geqq 0$ and for any $i .0 \leqq i \leqq 2 n-4$, let $\alpha_{i}(n, k)$ denote the number of cells of dimension equal to $i$ which are allowed in a $C W$ complex of dimension equal to $n-2$, which has the same homotopy type as $\mathbb{P}_{n-2}(\mathbb{C})-\widetilde{\Delta}_{n-2}$, and which is defined by recurrence. $\mathrm{CW}$ complexes which are (possibly) induced by $\widetilde{V}_{m}$ have index $k$ even.

THEOREM 1. For any $n \geqq 3$, for any $k \geqq 0$ and for any $i, 0 \leqq i \leqq n-2$,

$$
\alpha_{i}(n, k)=(n-1-i+k) \alpha_{i}(n-1, k)+(2 n-2-i+k) \alpha_{i-1}(n-1, k)
$$

and $\alpha_{i}(n, k)=0$ for any $i>n-2$.

We now give in the corollaries below some combinatioral consequences of this theorem. Here $C_{p, q}$ represents a binomial coefficient.

COROLLARY 1.1. For any $n \geqq 3$, for any $k \geqq 0$ and for any $i, 0 \leqq i \leqq n-2$,

$$
\alpha_{i}(n, k)=C_{n+k, i} \prod_{j=0}^{k}(n-1-i+j)(n-2) !(k+1) !
$$

COROLLARY 1.2. For any $n \geqq 3$ and for any $k \geqq 0$

$$
\sum_{i=0}^{n-2} \alpha_{i}(n, k)=(n-2) ! \sum_{i=0}^{n-2} 2^{i} C_{k+1+i, k+1}
$$

COROLLARY 1.3. For any $n \geqq 3$ and for any $k \geqq 0$

$$
\sum_{i=0}^{n-2} \alpha_{i}(n, k)=(-1)^{n}(n-2) !
$$

We write $A(t) \gg B(t)$ for any two polynomials $A(t), B(t)$ provided that $A(t)-B(t)=(1+t) C(t)$ where $C(t)$ has positive coefficients and the degree of $C(t)$ is one less than the maximum of the degrees of $A(t)$ and $B(t)$. Compare $A(t)>B(t)$ [2].

COROLLAR Y 1.4. For any $n \geqq 3$ and for any $k \geqq 0$

$$
\sum_{i=0}^{n-2} \alpha_{i}(n, k) t^{i} \gg \sum_{i=0}^{n-2} \beta_{i}(n) t^{i} .
$$


Here $\beta_{i}(n)=\operatorname{rank} H_{i}\left(\mathbb{P}_{n-2}(\mathbb{C})-\widetilde{\triangle}_{n-2}\right)$ for any $i, 0 \leqq i \leqq n-2$ and $H_{*}$ is integral singular homology. Corollary 1.4 gives sharp Morse-type inequalities between the cellular decomposition and the homology of $X_{m}$.

Let $\mu_{i}(n)$ denote a uniform greatest lower bound to the number of critical points of $\widetilde{V}_{m}$ with index equal to $2 n-4-i$ whenever $\widetilde{V}_{m}$ is a Morse function. We state an important consequence of Theorem 1.

THEOREM 2. For any $n \geqq 3$ and for any $i, 0 \leqq i \leqq n-2, \mu_{i}(n)=\alpha_{i}(n, 0)$ and $\mu_{i}(n)=0$ for any $i>n-2$.

COROLLARY 2.1. $\quad \sum_{i=0}^{n-2} \mu_{i}(n)=\left[2^{n-1}(n-2)+1\right](n-2)$ ! for any $n \geqq 3$.

This corollary bounds the number of critical points which $\widetilde{V}_{m}$ as a nondegenerate function has.

In [4] Smale asks whether this greatest lower bound on the distribution of types of critical points of $\widetilde{V}_{m}$ is ever realized. By a theorem [3] on the existence of degeneracies of $\widetilde{V}_{m}$ for some $\left(m_{i}\right) \in \mathbb{R}_{+}^{n}$ and for any $n \geqq 4$ the minimal classification is not always realized. For any three positive masses $\widetilde{V}_{m}$ is always a Morse function so that in this case the lower bound is always realized. Our main result follows.

THEOREM 3. For any $n \geqq 3$ there is a maximal open set in $\mathbb{R}_{+}^{n}$ for which the distribution of critical points of $\widetilde{V}_{m}$ as a nondegenerate function corresponds to the minimal classification given by $\Sigma \mu_{i}(n) t^{i}$. For any $n \geqq 4$ this open set is proper.

Remark. In [1] it is shown that this minimal classification exists as well on the boundary of the openset of Theorem 3, where degeneracies of $\tilde{V}_{m}$ occur, if we give a homology interpretation as in [1, Theorem 4]. The homology condition is closed for the minimal classification; therefore, the boundary of the open region is included.

\section{HOMOLOGY CLASSIFICATIONS}

Critical point theory may be applied to $\widetilde{V}_{m}$ for any $\left(m_{i}\right) \in \mathbb{R}_{+}^{n}$. This allows us to conclude that $\widetilde{V}_{m}$ always imposes a cellular decomposition of $\mathbb{P}_{n-2}(\mathbb{C})-\widetilde{\Delta}_{n-2}$. This decomposition must have $\Sigma \alpha_{i}(n, 0) t^{i}$ as a lower bound and for some $k \geqq 0, \Sigma \alpha_{i}(n, k) t^{i}$ must be an upper bound on the cellular decomposition given by $\widetilde{V}_{m}$. The upper bound is a uniform bound provided that it holds for each $\left(m_{i}\right) \in \mathbb{R}_{+}^{n}$. We need to assert only that such a uniform upper bound exists; i.e. that given any $n \geqq 3$, there is a $k_{n} \stackrel{\geqq}{=}$ such that $\Sigma \alpha_{i}\left(n, k_{n}\right) t^{i}$ bounds the cellular decomposition given by $\widetilde{V}_{m}$ for any $\left(m_{i}\right) \in \mathbb{R}_{+}^{n}$.

THEOREM 4. For any $n \geqq 3$ there exists a uniform upper bound on the number of critical points of $\widetilde{V}_{m}$ in each index $2 n-4-i, 0 \leqq i \leqq n-2$, for any $\left(m_{i}\right) \in \mathbb{R}_{+}^{n}$ for which $\widetilde{V}_{m}$ is nondegenerate. Let $\tilde{\mu}_{i}(n)$ denote this upper bound and let $\mu_{i}(n, m)$ denote the number of critical points of $\tilde{V}_{m}$ with index equal to $2 n-4-i$ whenever $\widetilde{V}_{m}$ is a nondegenerate function. Then $\Sigma \widetilde{\mu}_{i}(n) t^{i}>\Sigma \mu_{i}(n, m) t^{i}$. 
COROLLARY 4.1. $\Sigma \tilde{\mu}_{i}(n) t^{i}$ gives a uniform upper bound on the cellular decomposition arising from $\widetilde{V}_{m}$ for any $n \geqq 3$ and for any $\left(m_{i}\right) \in \mathbb{R}_{+}^{n}$. If $\Sigma \alpha_{i}(n, m) t^{i}$ denotes the cellular decomposition which corresponds to $\widetilde{V}_{m}$, then

$$
\Sigma \widetilde{\mu}_{i}(n) t^{i}>\Sigma \alpha_{i}(n, m) t^{i}
$$

Here we understand all sums above to range over $0 \leqq i \leqq n-2$.

Finally we give the relation between the cellular decomposition of $X_{m}$ by $\widetilde{V}_{m}$ and the relative homology of the critical set of $\widetilde{V}_{m}$.

For any $n \geqq 3$ and for any $\left(m_{i}\right) \in \mathbb{R}_{+}^{n}$ let $C_{1}<\ldots<C_{r}<0$ be the (finitely many) critical values of $\widetilde{V}_{m}$. Set $C_{0}=-\infty$ and for any $j, 1 \leqq j \leqq r$, let $W_{j}$ be defined by $\widetilde{V}_{m}^{-1}\left(C_{j-1}, C_{j}\right)$. Let $\Lambda_{j}$ be the set of critical points of $\widetilde{V}_{m}$ with critical value $C_{j}, 1 \leqq j \leqq r$. Finally, for any $i, 0 \leqq i \leqq 2 n-4$, define $\tau_{i}(n, m)$ by $\tau_{i}(n, m)=\sum_{i=1}^{r} \operatorname{rank} H_{2 n-4-i}\left(W_{j} \cup \Lambda_{j}, W_{j}\right)$. By the fact that the index of any critical point of $\widetilde{V}_{m}$ equals or exceeds $n-2$ [3], we have $\tau_{i}(n, m)=0$ for any $i>n-2$.

For any $n \geqq 3$ and for any $\left(m_{i}\right) \in \mathbb{R}_{+}^{n}$ let $\alpha_{i}(n, m)$ equal the number of cells of dimension $i$ in the CW complex which $\widetilde{V}_{m}$ by duality defines.

THEOREM 5. For any $n \geqq 3$ and for any $\left(m_{i}\right) \in \mathbb{R}_{+}^{n}$

$$
\sum_{i=0}^{n-2} \alpha_{i}(n, m) t^{i}>\sum_{i=0}^{n-2} \tau_{i}(n, m) t^{i}
$$

COROLLARY 5.1. For any $n \geqq 3$ and for any $\left(m_{i}\right) \in \mathbb{R}_{+}^{n}$ for which $\widetilde{V}_{m}$ has only nondegenerate critical points,

$$
\sum_{i=0}^{n-2} \alpha_{i}(n, m) t^{i}=\sum_{i=0}^{n-2} \tau_{i}(n, m) t^{i}
$$

\section{MINIMAL CLASSIFICATIONS}

It is easy to see that the minimal classification induced by $\Sigma \alpha_{i}(n, 0) t^{i}$ in the nondegenerate case, Theorem 2, or with the homology interpretation arises on a proper subset of $\mathbb{R}_{+}^{n}$ for any $n \geqq 4$. We refer to previous results [3] on the existence of degeneracies of $\widetilde{V}_{m}$ for some $\left(m_{i}\right) \in \mathbb{R}_{+}^{n}$ and for any $n \geqq 4$. Here there is a one parameter family of relative equilibria classes along which an index change occurs from maximum (index $=2 n-4$ ) to saddle (index $=2 n-6$ ) at a unique ratio of the masses. For certain choices of the masses, there is an excess of (at least) (n-2)! maxima over the number of maxima given by the minimal classification with either the nondegenerate or homology interpretation.

Let $\Sigma_{n}$ be the set of all $\left(m_{i}\right) \in \mathbb{R}_{+}^{n}$ for which there exists a degenerate relative equilibria class. Several possibilities arise. Let $U \subset \mathbb{R}_{+}^{n}-\Sigma_{n}$ be a maximal open set on which the minimal classification occurs. Then on the boundary of $U$, which is contained ni $\Sigma_{n}$, the homology minimal classification must arise. This is the essential closure property which is referred to in Section 2. However, 
it may happen that the homology minimal classification is realized for some $\left(m_{i}\right) \in \Sigma_{n}$ but does not occur locally in $\mathbb{R}_{+}^{n}-\Sigma_{n}$. Such is the case of $n=4$ masses [1]

In [3] we prove that $\Sigma_{n}$ has positive $(n-1)$ dimensional (Hausdorff) measure in $\mathbb{R}_{+}^{n}$ for any $n \geqq 4$. $\Sigma_{n}$ extends to the boundary of $\mathbb{R}_{+}^{n}$ in $\mathbb{R}^{n}$. This allows an induction to be carried out from $\Sigma_{n-1}$ to $\Sigma_{n}$. In this way, in particular, the minimal classification can be shown to exist on an open set in $\mathbb{R}_{+}^{n}$ for any $n \geqq 4$.

By comparing $\Sigma \mu_{i}(n) t^{i}$ with the Betti numbers $\Sigma \beta_{i}(n) t^{i}$ we find that the sharp Morse-type inequalities given by Corollary 1.4 show an excess of critical points in every dimension, $0 \leqq i \leqq n-2$. By comparing Corollary 2.1 and $\Sigma \beta_{i}(n)=n ! / 2$ [2] we find an excess of critical points equal to $\frac{1}{2}\left(2^{n}-n-1\right)(n-2)(n-2)$ ! for any $n \geqq 3$.

\section{REFERENCES}

1. Palmore, J.I., Bull. Am. Math. Soc. 81, $489-491$ (1975); MR 50, No. 15514.

2. Palmore, J.I., Letters Math. Phys. 1, 119-123 (1975).

3. Palmore, J.I., 'Measure of Degenerate Relative Equilibria, I', Annals of Math., to appear.

4. Smale, S., Manifolds-Amsterdam 1970 (Proc. Nuffic Summer School), Lectures Notes in Math., Vol. 197, Springer, Berlin, 1971, pp. 194-198; MR 43, No. 4429.

(Received November 1, 1976) 